\title{
Muséologies
}

Les cahiers d'études supérieures

\section{John R. Porter. Directeur général du Musée national des beaux-arts du Québec}

\section{Pierre-Olivier Ouellet}

Volume 2, numéro 2, avril 2008

URI : https://id.erudit.org/iderudit/1033587ar

DOI : https://doi.org/10.7202/1033587ar

Aller au sommaire du numéro

Éditeur(s)

Association Québécoise de Promotion des Recherches Étudiantes en

Muséologie (AQPREM)

ISSN

1718-5181 (imprimé)

1929-7815 (numérique)

Découvrir la revue

Citer ce document

Ouellet, P.-O. (2008). John R. Porter. Directeur général du Musée national des beaux-arts du Québec. Muséologies, 2(2), 30-40.

https://doi.org/10.7202/1033587ar d'utilisation que vous pouvez consulter en ligne.

https://apropos.erudit.org/fr/usagers/politique-dutilisation/ 


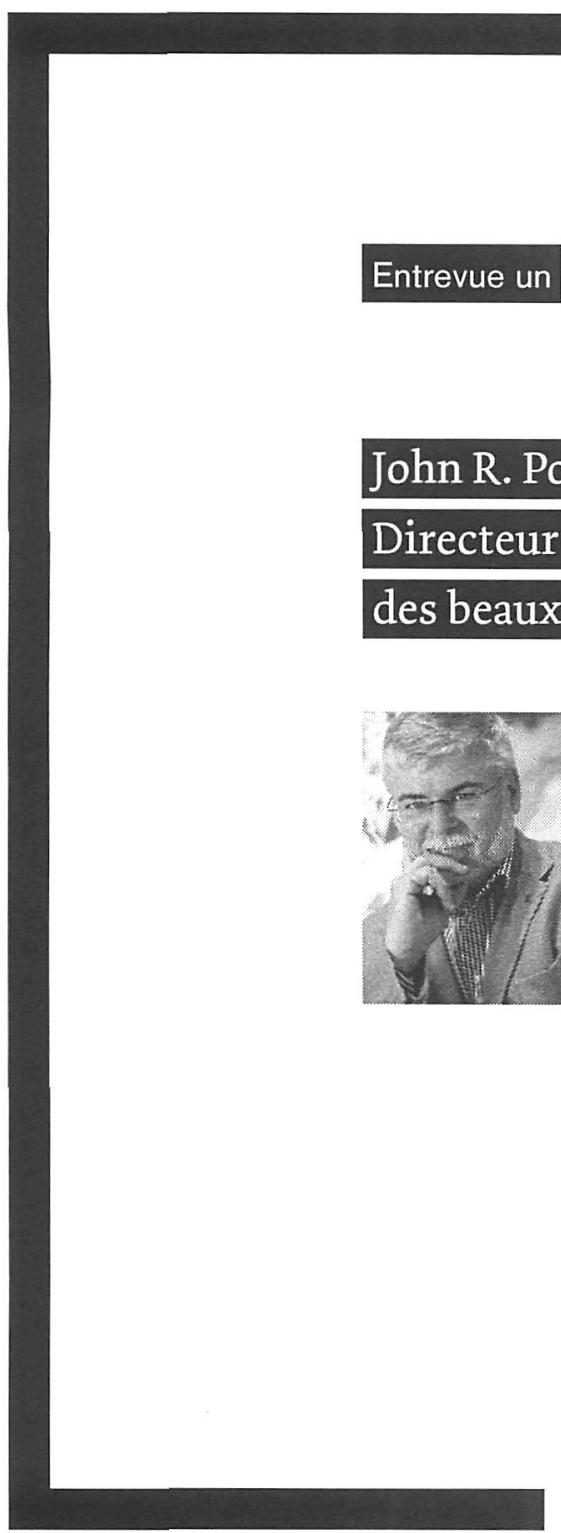

[réalisée par Pierre-Olivier Ouellet le 19 octobre 2007]

Historien de l'art de formation, John R. Porter a débuté sa carrière comme conservateur adjoint à la Galerie nationale du Canada (actuel Musée des beaux-arts du Canada, à Ottawa) de 1972 à 1978. Menant ensuite une double carrière dans le domaine des musées et de l'enseignement, il a été professeur à l'Université Laval, à Québec, et conservateur en chef du Musée des beaux-arts de Montréal. Depuis septembre 1993, John R. Porter est directeur général du Musée national des beaux-arts du Québec, à Québec. À ce poste depuis quinze années, il a enrichi la programmation par la tenue de nombreuses expositions mettant en valeur la production de plusieurs grands artistes québécois, comme le peintre Ozias Leduc (1996) ou le sculpteur Louis-Philippe Hébert (2001), et initié la présentation de grandes expositions comme Rodin à Québec (1998) et L'Univers baroque de Fernando Botero (2007).

Rappelons que le Musée national des beaux-arts du Québec, créé par le gouvernement du Québec, a ouvert ses portes en 1933. Après le nom de Musée de la Province (1933), de Musée du Québec (1966), l'institution a changé de dénomination en 2002 pour celle de Musée national des beaux-arts du Québec (MNBAQ). 
Pierre-Olivier Ouellet

Monsieur Porter, comment perceviez-vous le Musée du Québec à l'époque où vous étiez étudiant à l'Université Laval (licence et maîtrise)?

JOHN R. PORTER

J'étais très impressionné par le Musée du Quêbec. Dans les années 1960,j'ai visité des expositions sur Hartung (1969), Rouault (1965), Soulages (1968), Borduas (1967), de même que certaines expositions d'art traditionnel. Pendant ma licence,j'ai fait un seul cours surl'art au Québec. J'ai alors réalisé un travail de session avec Luc Noppen, sousl'égide de Jean Trudel, portant sur les églises de Charlesbourg et l'art traditionnel au Québec. On commençait à s'intéresser à ce type de patrimoine et c'était vraiment les rudiments. Je me souviendrai toujours d'avoir rencontréle directeur du musée de l'époque, Jean Soucy (directeurde 1967 à 1973). Il avait acceptéquel'on fasse une exposition qui avait duré quelques semaines dansl'une des salles du musée : Charlesbourg, atelier d'art traditionnel (1971). Cela a étéla toute première exposition de ma carrière. D'une certaine manière, le musée m'a donné ma première chance.

[1]

Notons que, en 2003, J. Porter a rédigé un essai dans la revue Archives, retraçant son parcours après dix années passées au MNBAQ. (PORTER, John R. "L'art de réinventer une institution nationale». Archives, vol. 34, no 3 , 2003, p. 73-88)
Pierre-Olivier Ouellet

Vous avez annoncé, en 2007, que vous quittiez la direction du MNBAQ. Vous aurez complété quinze années à la tête de l'institution, réalisant ainsi le plus long directorat depuis Gérard Morisset (1898-1970), directeur de 1953 à 1965. Comment analysez-vous votre directorat ${ }^{[1]}$ ?

\section{JOHN R. PORTER}

Mes quinze années à la barre du musée m'ont permis de mener de grands chantiers à terme, de sécuriser l'institution, de lui donner une stabilité, de lui assurer un continuum. L'institution n'est pasle fait d'un individu, mais d'une équipe, notamment du noyau de directeurs qui se sont succédés et dont la chimie produit ses effets. Pour qu'il y ait des collaborateurs de toutes parts à une exposition comme Québec, une ville et ses artistes (2008), en plus de la participation de trois générations différentes d'historiens de l'art, il fallait nécessairement qu'il $y$ ait un continuum.

De manière générale, je dirais également que j'ai eu trois étapes de direction. Il y a eu d'abord une période de sauvetage. Il était nécessaire de redonner confiance et d'assurer une notoriété au musée, de lui tailler une place sur la scène publique. Cela a été difficile, notamment en raison des résistances au sein même del'organisation..., mais cela fait partie du jeu.J'ai pensé un certain temps à quitter le musée puis, un bon matin, j'ai pris la décision de rester en dépit des difficultés, même si je gagnais à peine un peu plus qu'un professeur d'université; j'ai décidé de persévérer. 
Cela s'est soldéparl'exposition Rodin à Québec (1998) ${ }^{[2]}$, marquant une période d'affirmation et defort positionnement institutionnel, de rattrapage et de réseautage international, etc. Ce qui s'est évidemment poursuivi depuis 1998. Enfin, depuis cinq ans, tout en continuant le boulot, avec une vision du réel, je suis davantage tourné versl'avenir, préparant le terrain pour celui ou celle qui va me suivre à la direction du musée. D'ailleurs, il y a pour moi une ironie incroyable dans ce que je vis présentement. J'ai annoncéau mois de mars 2007, après mûre réflexion, que je quittais la direction. Il était clair alors qu'en septembre 2007 tout devait être réglé. J'avais planifié toute une programmation, un peu du type "feux d'artifice". On le voit d'ailleurs pour cet hiver et pour l'été 2008, avec les expositions Québec, une ville et ses artistes, puis Le Louvre à Québec. Les arts et la vie. Ces événements majeurs étaient normalement destinés à ma ou mon successeur. En arrivant en poste, non seulement elle ou il aurait une planification déjà prête pour les trois années à venir, mais également des expositions très solides en tout début de mandat.

[2]

Sur l'importance de l'exposition Rodin à Québec, qui a attiré 524273 visiteurs au cours de l'été 1998, consulter PORTER, John R. «Rodin, Laliberté et les autres. Réalités et perception d'un musée d'art'. Annales d'histoire de l'art canadien, vol. 20, nos 1-2, 1999, p. 205-221.
Étant donnéque l'on cherche encore un nouveau directeur général, et c'est bien là qu'est l'ironie, j'aurai finalement été présent pour inaugurer ces expositions. Mais, en même temps, le plaisir pour un directeurn'est-il pas d'initier des projets en sachant qu'ils vont se réaliser?

\section{Pierre-Olivier Ouellet}

Depuis votre entrée en fonction en 1993, on s'apercoit que le musée est parvenu à changer d'image, passant d'une reconnaissance régionale à internationale. Comment cette transformation s'est-elle produite?

JOHN R. PORTER

C'est par ce type de bilan que l'on remarque que le temps est important. Le musée, c'est une construction. Vous savez, lorsquej'y suis arrivé, un des grands défis que je devais relever était le fait que l'institution n'était même pas au niveau du sol, mais dans le trou... et il fallait sortir du trou! La programmation prévue ne couvrait que les six premiers mois; ensuite, il n'y avait plus rien. Les caisses étaient vides. Le budget avait été absorbé par la réouverture (en 1991, à la suite de l'agrandissement amorcé en 1988, intégrant l'ancienne Prison de Québec au complexe muséal). Je devais donc apprendre à m'occuper de tout et à reconsidérer l'institution. 
Et, à l'époque, je ne m'en suis jamais caché, il n'y en avait que pour le Musée de la civilisation (à Quebec), dont le budget d'une exposition moyenne correspondait à celui quej'avais pour l'ensemble des expositions de l'année. Il était facile de nous dire "vous n'êtes pas populaire", ce qui me mettait en rogne! J'ai fait quelques sorties publiques, pas trop... parce que les gens n'aiment pas les râleurs. Il fallait plutôt trouver le moyen de «livrer». C'est pour cette raison que l'exposition Rodin a été aussi extraordinaire. Elle constituait une immense opération à risque, alors que nous n'avions niles moyens de la présenter, ni de support spécial du gouvernement. Nous avons donc réalisé cette exposition en mettant sur une case de l'échiquier le budget de toutes les expositions de l'année, de toutes les acquisitions et de toute la formation. Tout, environ 1,1 million de dollars, a été placé surce seul événement ! Et, heureusement, ça a marché! Un gestionnaire doit être capable de prendre des risques calculés. J'ai personnellement renoncé à ma sécurité d'emploi à l'Université Laval; je ne suis pas fonctionnaire. Si mon contrat n'est pas renouvelé, c'est fini. En même temps, le fait d'être directeur $\mathrm{du} M N B A Q$ procure une liberté extraordinaire, tant que l'on est confiant et que l'on est capable de vendre ses projets, de convaincre. La conviction et la détermination sont des outils essentiels.
Pierre-Olivier Ouellet Et quels autres outils ont façonné votre pratique comme directeur général ? Qu'avez-vous appris au cours de cette pratique?

\section{John R. PORTER}

L'écrit est ce qui m'a aidé dans mon métier d'historien de l'art, de muséologue et de directeur général. Parce quel'écrit m'a forcéa fixer ma pensée, à garder des traces de ce quej'ai fait, et a abouti éventuellement à une contribution comme historien del'art. Voyez,je vais vous montrer ceci (M. Porter présente un petit cahier vert à reliure spirale, légèrement épaissi par quelques papiers collés sur certaines des pages). Je n'appellerais pas cela mon journal de bord, mais c'est mon quotidien $d u$ (en lisant une note inscrite sur la couverture du cahier) lundi 26 novembre 2007 au 18 février 2008. Depuis que je suis au musée, j'en ai rempli ça de long (M. Porter étire les bras d'un geste ample). Chaque jour, je prend en note ce quej'ai fait, qui j'ai rencontré, pourquoi, ce dont on a discuté, ainsi de suite, sans entrer dans une dynamique d'étalage de sentiments. Dans deux ans, trois ans, dix ans, on pourra me poser une question sur ce qui s'est passé et je regarderai dans mes notes pour retrouver l'elément et me remémorer les faits. C'est le cursus qui a étéle mien. 
Il est aussi essentiel d'apprendre à travailler sur plusieurs dossiers à la fois. Il est inouï de constater tout ce quel'on peut alors amasser, parce que, en ayant plusieurs dossiers, on tombe surdes choses au hasard qui, peu à peu, enrichissent les divers projets. De sorte que, au moment de travailler plus attentivement sur l'un des dossiers, celui-ci est déjà pas mal nourri, il a une consistance. Plus d'une foisj'ai été sauvé par des notes écrites un samedi matin et simplement déposées dans un dossier. Souvent, il en ressort les plus beaux textes quel'on rédige par rapport à tel ou tel essai qu'on avait promis. Il faut savoir saisir ces moments d'écriture au cours desquels on noircit une feuille ou deux. Pour moi, c'est ma soupape, ma courroie de sécurité.

Ensuite, je vous dirais que le détail est un danger, qu'il constitue un mauvais refuge pour un directeur général. Il devient un prétexte pour ne pas faire et ne pas se commettre, alors que l'on doit livrer ce qui a été promis. On est dans une discipline qui avance et on doit toujours se commettre. Il faut accepter que l'on puisse en partie se tromper et se dire tout de même qu'on a fait une avancée importante : des pistes seront ouvertes et d'autres personnes pourront éventuellement prendre le relais. Tant mieux si le travail est aussibon que possible. Mais, quand on s'éternise sur un projet, quel'on se complaît dans un certain maniérisme, on se prive du plaisir que l'on aurait à investir d'autres champs, d'autres secteurs. Cela ne veut pas dire qu'il faille bâclerle travail, mais il $y$ a un temps pour commencer et un temps pour finir.

Aussi, il ne faut pas faire la vie comme on voudrait qu'elle soit, il faut la prendre pour ce qu'elle est. Ce n'est pas parce que telle année on a le goût de présenter telle exposition qu'on peut le faire à ce moment-là. Il est important de la garder à l'esprit, même d'en rêver. Il faut toutefois "atterrir", garder les pieds sur terre. Une bonne façon d'y arriver, c'est de pratiquer le "coup retenu", de ne pas insister quand ce n'est pas le bon moment. Quand le projet est mûr, il se réalise. On peut $y$ travailler, mais cela fonctionne généralement de la sorte, tant en matière d'acquisition que d'exposition. Il ne faut donc pas tirer sur la fleur pour la faire pousser plus vite.

Par exemple, il y a cinq ans, je rêvais de présenter Botero après avoir visité à Paris une exposition de l'artiste au Musée Maillol Fondation Dina Vierny.J'avais essayé de l'obtenir pour Québec, mais c'était compliqué... puis cela n'a pas marché. Un an plus tard,j'en ai parlé avec des collègues; c'était encore laborieux et trop onéreux. Puis, pendant l'exposition Camille Claudel et Rodin (2005), j'ai entendu parler que Art Services International aux États-Unis avait un projet Botero. À la fin de l'été,je savais que nous aurions une exposition, en plus d'être les premiers à la présenter. Dès que le projet a été confirmé, j'ai contacté Fernando Botero 
et je me suis assuré qu'il viendrait à Québec. L'inauguration de notre exposition (L'Univers baroque de Fernando Botero, 2007) a constituéla seule étape où l'artiste est allé en personne. L'organisation d'une exposition est un processus ancré dans une longue durée et, en même temps, cet exemple démontre qu'il faut demeurer ouvert et attentifà ce qui passe.

Enfin, ce qui est important aussi dans ce type de travail, c'est la vision. Le mot est galvaudé, mais c'est bien de la vision dont il s'agit. Sinon, on peut se laisser prendre dans la spirale du quotidien : brasser beaucoup de choses, beaucoup de papiers, aller dans toutes les directions, terminer sa semaine en poussant un soupir de soulagement... et, qu'est-ce qu'on a fait? Dans la gestion même du travail, il faut poursuivre de grands objectifs pour éventuellement atteindre de grands objectifs. J'aime beaucoup citer le cas de Paul Rainville (1887-1952) qui, pour moi, a probablement étéle plus grand directeur du musée (1941-1952). Il était une personne de vision, d'innovation, qui possédait de grandes habiletés en communication. Au debbut des années 1940-1950, il a introduit les programmes éducatifs, la musique au musée, des expositions itinérantes, des expositions internationales avec catalogue, des appariements avec l'École des beaux-arts. Il a même écrit un texte, demeuré inédit, sur le musée et l'éducation des adultes au Canada en 1951. Mais qui était Paul Rainville? C'était un grand vendeur d'assurances, parfaitement bilingue. Il a dû mettre un terme à cette première carrière après avoir souffert de la tuberculose. Il était le beau-frère de LouisAthanase David (1882-1953), secrétaire de la province sous le gouvernement libéral de Louis-Alexandre Taschereau (1867-1952), soit l'équivalent aujourd'hui du ministre de la Culture et des communications. En 1933, année d'ouverture du Musée, Paul Rainville a écrit un grand article rendant hommage à Taschereau. De fil en aiguille, il est devenu l'adjoint de PierreGeorges Roy (1870-1953), le deuxième directeurdu musée (1932-1941). Il a pris en charge tout le volet des sciences naturelles, puis le volet artistique. Il était un visionnaire, très proche des artistes, avec une belle force de conviction. Sensible aux besoins de son personnel, il a travaillé dans des périodes de crise (économique des années 1930) et de guerre (Seconde Guerre mondiale). Il n'y avait pourtant rien qui annonçait que Paul Rainville parviendrait à faire autant pour l'institution... surtout parle type de canal qu'il avait emprunté pour entrer au musée. Pourtant, il a réalisé des merveilles. 
Pierre-Olivier Ouellet

Est-ce que le fait d'être historien de l'art de formation et spécialiste de l'art québécois vous a permis de mieux diriger une institution muséale dont la mission est «de faire connaître, de promouvoir et de conserver l'art québécois de toutes les périodes ${ }^{[3]}$ ?

\section{JOHN R. PORTER}

D'avoir été un historien de l'art "patenté" pour remplir la fonction de directeur général du MNBAQ c'était parfait, surtout au moment où je suis entré en fonction en 1993. À cette époque, pour nos grands noms, bien souvent, il n'y avait eu aucune exposition appréciable. Tout un travail a été mené, axé sur ce j'appellerais les "grandes expositions de rattrapage». En raison de ma spécialisation, l'avantage est que certaines questions ne se posaient pas : il était évident qu'il fallait exposer tel ou tel artiste. Généralement, une fois la décision prise, je constatais que je ne m'étais pas trompé. Parfois oui... mais pas souvent. Cette réalité d'historien de l'art a coloré mes orientations comme administrateur.

[3]

Loi sur les musées nationaux, L.R.Q., chapitre M-44. [4]

Lettre de Phyllis Lambert adressée à MM. Pierre Lassonde et John R. Porter, datée du 14 janvier 2008. Rendue publique quelques jours plus tard, la courte lettre a notamment été citée par Antoine Robitaille ( Phyllis Lambert s'insurge contre la démolition du couvent des Dominicains". Le Devoir, mardi 22 janvier 2008).

\section{Pierre-Olivier Ouellet}

Le 20 décembre 2007, un communiqué des gouvernements fédéral et provincial annonçait leur participation financière à un nouveau projet d'agrandissement du musée, assurant sa concrétisation pour 2013. Un nouveau pavillon vous permettra d'exposer davantage la collection (à peine $2 \%$ est exposé actuellement), d'ouvrir un espace adapté à l'art de la relève et aux nouvelles technologies, de mieux accueillir le public qui fréquente de plus en plus l'institution, etc.

Pierre Lassonde, président du conseil d'administration du MNBAQ, a déjà financé l'achat du site où sera édifié le nouveau pavillon. Il s'agit de l'ancien site des Dominicains, où se trouvent notamment une église, un presbytère et un couvent, construits entre 1918 et 1930 selon les plans de l'architecte JosephAlbert LaRue (1891-1985). La démolition annoncée du couvent des Dominicains a soulevé plusieurs contestations, tant des résidents du quartier du musée que des spécialistes en architecture et en urbanisme. Phyllis Lambert, directrice fondatrice du Centre Canadien d'Architecture, croit que cette «proposition de départ envoie un message négatif vis-à-vis de la conservation du patrimoine" [4]. Elle propose d'intégrer le couvent à l'architecture du nouveau pavillon. Que répondez-vous aux détracteurs du musée?

JOHN R. PORTER

Qu'ils ont présumé que nous étions des irresponsables. Pourtant, nous réfléchissons depuis cinq ans à la question. Nous consultons et nous respectons le travail qui a été accompli par des historiens del'art et de l'architecture qui œuvrent pour le ministère de la Culture et des communications et pour la Ville de Québec. Nous avons commandé des études à des architectes. Il y a eu tout un travail préparatoire. 
Nous n'agissons pas de manière inconséquente. Nous avons exploré le site des Dominicains. À un moment donné, ces derniers sont venus frapper à notre porte en nous demandant : "Seriez-vous intéressés, parce que nous préférons avoir un établissement culturel plutôt que des condominiums. "Nous avons conclu une entente. Nous ne les chassons pas, ils désirent partir. Pour l'édifice proprement dit, et comme je l'ai écrit à Phyllis Lambert, selon les propres propos des Dominicains le monastère est un immeuble qui "s'en va chez le diable». Conserver la structure, comme elle le propose, ne permettrait de satisfaire que $17 \%$ des besoins de nouveaux espaces du musée (avec le nouveau pavillon, il est plutôt prévu de doubler la superficie disponible pour les expositions). Nous ne sommes tout de même pas irresponsables. Il n'y a pas de mépris dans nos décisions et nous n'allons pas raserl'ensemble des bâtiments associés au passage des Dominicains, un ensemble qui, incidemment, n'est pas classé. Nous conserverons notamment le presbytère auquel la communauté et la paroisse sont attachés. Cela dit, qu'est-ce qui nous empêche d'avoir un nouveau repère dans la cité, un nouveau pavillon franchement $X X I^{e}$ siècle, qui sera un geste de création? Ma vision, c'est le musée de demain. Nous n'enfermerons tout de même pas la création contemporaine et les jeunes artistes dans un couvent vétuste et déserté! Il faut plutôt établir un musée digne de ce nom.
Par ailleurs, si aujourd'hui on disait et cela renverserait tous mes critères ! - que c'est un bâtiment très important pour le patrimoine de Québec, cela ne serait pas compliqué. Quand le MNBAQ a négocié avec l'Industrielle Alliance, celle-ci s'est retirée du dossier pour favoriser notre projet culturel. S'il advenait que le musée ne puisse pas réaliser son projet, l'entreprise s'est gardé un droit de premier refus. Théoriquement, si le musée se retire, un promoteur arriverait rapidement et installerait des condominiums surle site. Nous n'y gagnerions rien. À l'inverse, nous voulons inscrire notre projet dans une dynamique de long terme. Non seulement en assurant la conservation du batiment du presbytère, le seul a avoir conservé son intégrité architecturale, mais également par un engagement à long terme concernant l'église. Dans la situation actuelle du patrimoine religieux, qu'un musée d'État s'engage déjà dans la prise en charge d'un édifice religieux est l'un des plus beaux gestes patrimoniaux qui soit. Je ne comprends donc pas que certains s'en offusquent. Et quand on me dit que le monastère est un bâtiment centenaire, je réponds que ce n'est pas le cas. Construit dans les années 1920-1930, c'est un pauvre battiment truffé d'amiante et d'infiltrations, qui a vécu un incendie (1939), qui a été " trafiqué» et qui n'a plus rien à voir avec la conception d'origine de son architecte, Joseph-Albert LaRue.

Plus encore, cela pose la question fondamentale du patrimoine. En cette matière, il faut savoir choisir. Tout ne peut pas être conservé. 


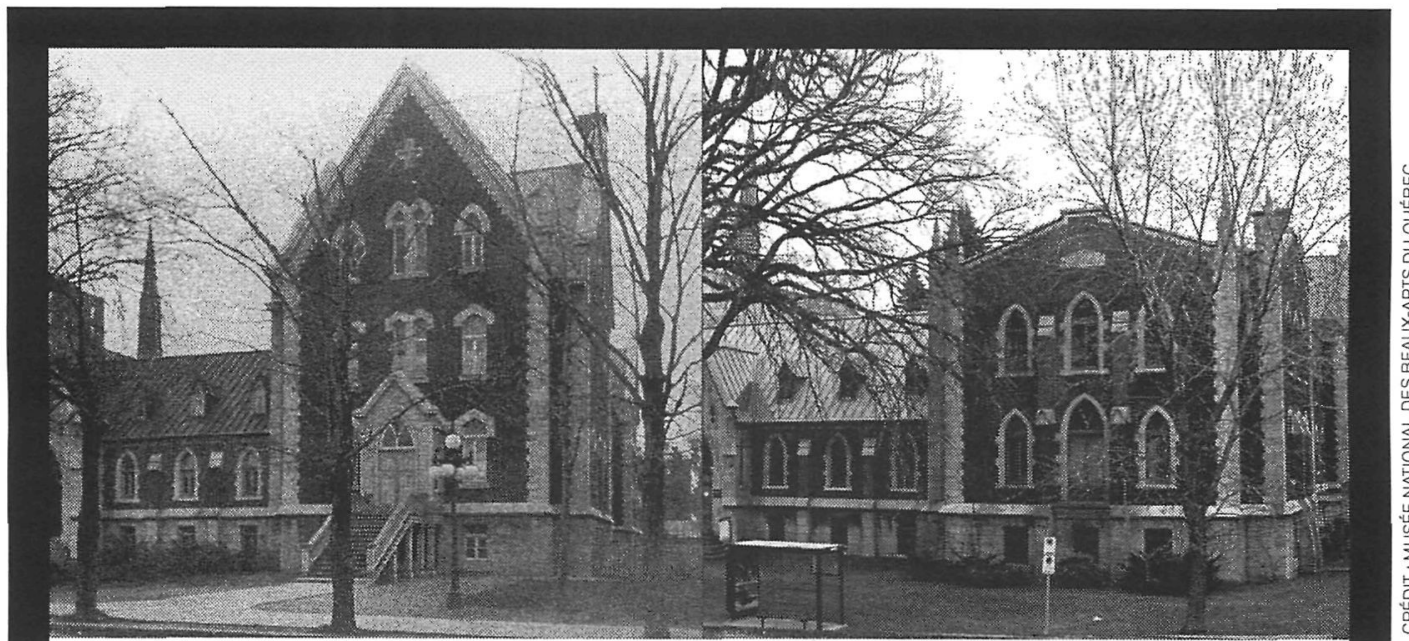

FIGURES 1 ET 2

Couvent des Domincains avant et après l'incendie. À la suite de l'incendie de 1939, l'aile ouest du couvent a perdu un étage, ses combles, son toit à deux versants et les nombreuses lucarnes qui faisaient son charme.

nous avons toutefois le devoir de l'excellence. Par exemple, lorsque le musée acquiert une œuvre, il faut qu'elle soit importante. Il m'est déjà arrivé, comme directeur ou conservateur, de ne pas retenir un tableau d'Antoine Plamondon (1804-1895) offert au musée. Pourtant, c'est un artiste très important! Mais s'il y a déjà dans les collections quelque chose de plus fort, de plus conséquent, si l'œuvre n'apporte rien de majeur, si nous savons qu'elle va rester dans les réserves et que même au jour d'une rétrospective l'œuvre ne figurera pas dans l'exposition, nous n'avons pas le droit d'investir des fonds et d'utiliser de l'espace de conservation pour des centaines d'années pour une œuvre qui n'est pas pertinente. En matière de patrimoine immobilier, c'est la même chose. Nous avons des devoirs et il serait irresponsable d'investir dans quelque chose qui ne le mérite pas pleinement. Nous devons être responsables maintenant puisque demain nous devrons nous demander ce que nous ferons collectivement par rapport à des ensembles immobiliers très impor- tants, comme celui des Ursulines de Québec, soit un ensemble extraordinaire qui remonte au régime français et qui, aujourd'hui, est au cœur de notre identité. Allons-nous y préférer un édifice des années 1920-1930 qui est passé au feu? C'est une question de choix. Vous savez, vouloir tout sauver c'est accepter de renoncer à tout; sous prétexte de responsabilité, c'est poser la pire des irresponsabilités. Il faut donc apprendre à choisir. Surce plan, il reste encore tout un travail d'éducation et d'explication à effectuer pour faire comprendre cette réalité. 


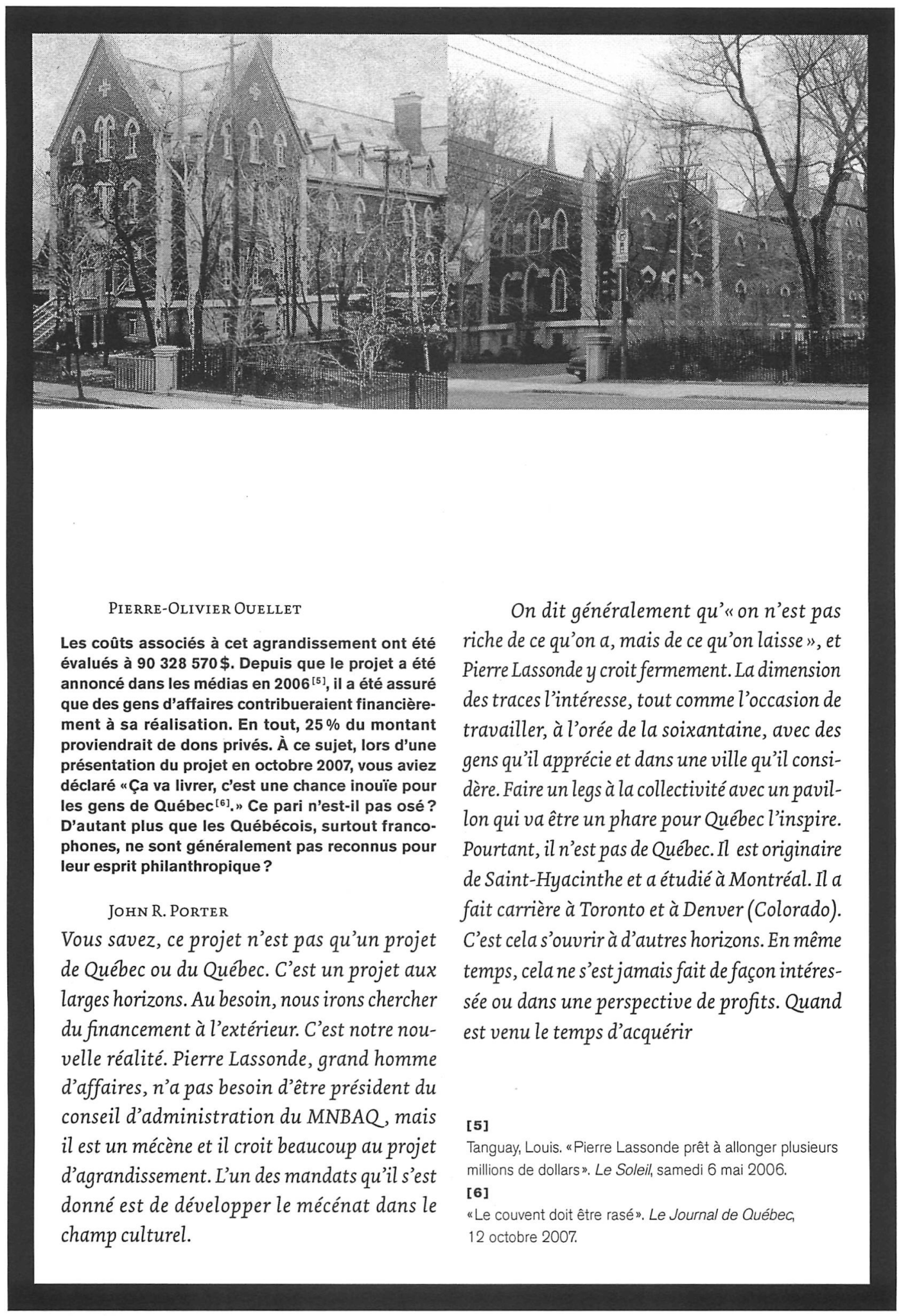


le site des Dominicains et de verser une compensation à l'Industrielle Alliance, Pierre Lassonde a simplement mis l'argent sur la table. Moimême, lorsquej'aurai quittéla direction, je vais ensuite m'occuper de la fondation du MNBAQ. Je ne suis pas un promoteur immobilier.Je veux seulement laisser, à ma manière, quelque chose à une ville que j'aime beaucoup, à une collectivité artistique, etc. J'ai même prévu, comme point de départ, de travailler à la fondation à titre bénévole. C'est important d'en parler, puisqu'on a presque fini, avec les années, par déconsidérer certains types d'engagement. Il faut ramener ce qu'il y a de beau dans le mécénat. Et s'il y a une reconnaissance, ou des tributs d'estime, tant mieux. On ne peut tout de même pas faire les choses en se cachant ! On est aux antipodes de l'intérêt et, également, on est dans la dynamique où l'on cherche à être exemplaire: faire des choses de la belle façon, inspirante pour les tiers qui vont suivre.

Pierre-Olivier Ouellet

Pour conclure, un concours international d'architecture devrait être ouvert dans les prochains mois. Quelle image le nouveau pavillon du musée devrait-il projeter au public?

JOHN R. PORTER

L'ouverture! Ouverture sur le quartier, sur la ville, surle Québec, surle monde. Avoirl'esprit ouvert. Un lieu d'invention. Il doit donner le signal d'une ouverture et non d'un mur et d'une fermeture. De manière symbolique, on pourrait dire que la prison projetait l'image d'un enfermement, d'un blockhouse. Maintenant, on veut ouvrir. C'est une tout autre philosophie. On ne travaille pas que pour des œuvres, on travaille d'abord pour ceux et celles qui permettent au musée d'exister et d'accomplir sa mission, c'està-dire les artistes et le public.

Le musée va donc sortir de son enclave, de son écrin de verdure, de son îlot provincial sur un terrain fédéral, brisant la distance qui le sépare du public. Il faut s'approcher tout en tenant compte des racines et en restant sensible à cette dynamique nature/culture. C'est magique, ce que l'on peut faire. Il y a tout à inventer pour ce nouveau pavillon, tant dans son architecture que dans le redéploiement des collections qu'il implique. L'espace va accueillir des œuvres que, vous et moi, nous ne soupçonnons même pas. À cet égard,j'espère que je serai personnellement surpris à l'ouverture du nouveau pavillon dans cinq ans. Agréablement surpris! 